\title{
Integration of gold nanoparticles in optical resonators
}

\author{
Alberto Jiménez-Solano, ${ }^{1}$ Carmen López-López, ${ }^{1}$ Olalla Sánchez-Sobrado, ${ }^{1}$ José Miguel Luque, ${ }^{1}$ \\ Mauricio E.Calvo, ${ }^{1}$ Cristina Fernández-López, ${ }^{2}$ Ana Sánchez-Iglesias, ${ }^{2}$ Luis M. Liz-Marzán, ${ }^{2}$ Hernán \\ Míguez $^{1, *}$
}

1. Instituto de Ciencia de Materiales de Sevilla, Consejo Superior de Investigaciones CientíficasUniversidad de Sevilla, C/América Vespucio 49, 41092 Sevilla, Spain.

2. Departamento de Química Física, Universidad de Vigo, 36310 Vigo, Spain

hernan@icmse.csic.es

RECEIVED DATE: $3^{\text {st }}$ January 2012

\begin{abstract}
The optical absorption of one-dimensional photonic crystal based resonators containing different types of gold nanoparticles is controllably modified by means of the interplay between planar optical cavity modes and localized surface plasmons. Spin-casting of metal oxide nanoparticle suspensions was used to build multilayered photonic structures that host (silica-coated) gold nanorods and spheres. Strong reinforcement and depletion of the absorptance was observed at designed wavelength ranges, thus proving that our method provides a reliable means to modify the optical absorption originated at plasmonic resonances of particles of arbitrary shape and within a wide range of sizes. These observations are discussed on the basis of calculations of the spatial and spectral dependence of the optical field intensity within the multilayers.
\end{abstract}




\section{Introduction}

One of the most exciting platforms to study photonic effects at the nanoscale is that of metal colloids, which display strong localized surface plasmon resonances. ${ }^{1}$ Research carried out in the last two decades has led to a wide diversity of synthetic methods that allow obtaining nanoparticles of precisely controlled composition, size and shape, which in turn yields fine tuning of their optical absorption and scattering properties. ${ }^{2-4}$ Also, dielectric layers of well-defined thickness and composition can be grown covering the metal particles, providing an alternative way to modify their extinction spectra. ${ }^{5}$ Besides these widespread methods, some attempts have been done to tailor the absorption of metal particles

through the interplay between photonic and plasmonic resonances. ${ }^{6-14}$ In this regard, most efforts have focused on the infiltration of metal colloids within two- or three-dimensional periodic dielectrics. Although modification of the photonic crystal properties of the ensemble by the presence of metal particles has been observed, no significant modification of the actual optical absorption has been reported. In this context, our groups have recently demonstrated that the absorptance of onedimensional photonic crystal based structures containing gold particles in different configurations can be strongly modified by such an interplay. ${ }^{15}$ In that work, $\mathrm{SiO}_{2}$ and $\mathrm{TiO}_{2}$ nanoparticle suspensions were alternately deposited to create a multilayer whose periodicity was interrupted by the presence of a layer of silica coated gold nanospheres $\left(\mathrm{Au} @ \mathrm{SiO}_{2}\right)$, whose size (diameter $\left.d<45 \mathrm{~nm}\right)$ and shape were similar to those of the pure $\mathrm{SiO}_{2}$ particles already present in the structure. This allowed them to be readily integrated in the multilayer. However, the applicability of this technique to tailor the absorption of ensembles containing metallic particles of arbitrary size and shape remains to be proved.

Herein we demonstrate that nanoparticle based multilayers are versatile structures to incorporate metal particles of large size and/or asymmetric shape. As a proof of concept, we report on the preparation and optical characterization of multilayered structures with optical cavities that incorporate large $\mathrm{Au} @ \mathrm{SiO}_{2}$ spheres (113 nm core plus $35 \mathrm{~nm}$ coating, total diameter $d \approx 183 \mathrm{~nm})$ and rods $(92 \mathrm{~nm} \times 47 \mathrm{~nm}$, with a gold core of $60 \mathrm{~nm} \times 15 \mathrm{~nm}$ ). In both cases, structures with high optical quality have been built. In 
order to achieve so, careful choice of the spin coating final rotation speed to deposit both the metal particle and the surrounding dielectric layers is a must, in order to minimize the effect of the structural defects introduced by the presence of such comparatively large beads. We prove that the absorption due to the respective localized surface plasmon resonances can be tailored to measure by means of the interplay with multiple photonic resonances.

\section{Experimental}

\section{Synthesis of Au@SiO particles}

Tetrachloroauric acid $\left(\mathrm{HAuCl}_{4} \cdot 3 \mathrm{H}_{2} \mathrm{O}\right)$, tetraethylorthosilicate (TEOS) and $\mathrm{NH}_{4} \mathrm{OH}(29 \%)$ were purchased from Aldrich. Ascorbic acid, sodium citrate $\left(\mathrm{C}_{6} \mathrm{H}_{5} \mathrm{O}_{7} \mathrm{Na}_{3} \cdot 2 \mathrm{H}_{2} \mathrm{O}\right)$, silver nitrate $\left(\mathrm{AgNO}_{3}\right)$ and sodium borohydride $\left(\mathrm{NaBH}_{4}\right)$ ) were supplied by Sigma. Cetyltrimethylammonium bromide (CTAB) and $O$-[2-(3-mercaptopropionylamino)ethyl]- $O^{\prime}$-methyl-poly(ethylene glycol) (mPEG-SH, Mw 5000) were procured from Fluka. $\mathrm{HCl}$ (37\%) was supplied by Panreac. All chemicals were used as received. Pure-grade ethanol and Milli- Q-grade water were used in all preparations.

Au nanospheres were synthesized by a previously reported method. ${ }^{16}$ Citrate-stabilized Au seeds $(\mathrm{d}=15$ $\mathrm{nm}, 0.5 \mathrm{M}$ ) were prepared by citrate reduction. ${ }^{17}$ In order to prepare large Au spheres, two growth steps were needed. In the first stage, Au seeds, in a final concentration of $3.9510^{-6} \mathrm{M}$, were added to a mixture of $\mathrm{HAuCl}_{4}(0.25 \mathrm{mM}), \mathrm{CTAB}(0.015 \mathrm{M})$ and ascorbic acid $(0.5 \mathrm{mM})$ at $37^{\circ} \mathrm{C}$; the nanoparticles synthesized in this way were employed as seeds in the following step. Large Au spheres were obtained by means of the same procedure using the last seed in a final concentration of $3.44 \cdot 10^{-5} \mathrm{M}$. Au nanorods were prepared by Ag-assisted seeded growth. ${ }^{18}$ The seed solution was prepared by borohydride reduction of $5 \mathrm{~mL} \mathrm{HAuCl} l_{4}(0.25 \mathrm{mM})$ in a $0.1 \mathrm{M}$ aqueous CTAB solution. The seed solution was added to a growth solution containing $0.1 \mathrm{M} \mathrm{CTAB}, 0.5 \mathrm{mM} \mathrm{HAuCl}_{4}, 0.019 \mathrm{M} \mathrm{HCl}, 0.8 \mathrm{mM}$ ascorbic acid and $0.12 \mathrm{mM}$ silver nitrate. Upon replacement of nanoparticle ligands by $\mathrm{mPEG}-\mathrm{SH},{ }^{19}$ the Au particles were transferred into ethanol, where silica coating was carried out through addition of TEOS and ammonia in the following final concentrations, $[\mathrm{Au}]=0.5 \mathrm{mM},\left[\mathrm{H}_{2} \mathrm{O}\right]=10.55 \mathrm{M},\left[\mathrm{NH}_{3}\right]=0.2 \mathrm{M}$, 
$[$ TEOS $]=0.8 \mathrm{mM}$. The core diameter and shell thickness were $113 \pm 11 \mathrm{~nm}$ and $35 \pm 7 \mathrm{~nm}$ for spheres, respectively, and $61 \pm 7 \times 16 \pm 1 \mathrm{~nm}$ and $16 \pm 3 \mathrm{~nm}$ for rods.

\section{Synthesis of $\mathrm{TiO}_{2}$ particles}

$\mathrm{TiO}_{2}$ nanoparticulated sols were synthesized using a procedure based on the hydrolysis of titanium tetraisopropoxide $\left(\mathrm{Ti}\left(\mathrm{OCH}_{2} \mathrm{CH}_{2} \mathrm{CH}_{3}\right)_{4}, 97 \%\right.$ Aldrich, abbrev. TTIP) as previously described. ${ }^{[22]}$ Briefly, TTIP was added to Milli-Q water, the white precipitate filtered and washed several times with distilled water. The resulting solid was peptized in an oven at $120^{\circ} \mathrm{C}$ for 3 hours with tetramethylammonium hydroxide (Fluka). Finally, the suspension was centrifuged at $14,000 \mathrm{rpm}$ for $10 \mathrm{~min}$. $\mathrm{SiO}_{2}$ nanocolloids were purchased from Dupont (LUDOX TMA, Aldrich). Both suspensions were diluted in methanol with concentrations of 4 wt.\% $\left(\mathrm{TiO}_{2}\right)$ and 3 wt.\% $\mathrm{SiO}_{2}$ and then using syringe driven filters (Millipore) of $0.22 \mu \mathrm{m}$ and $0.45 \mu \mathrm{m}$ for $\mathrm{TiO}_{2}$ and $\mathrm{SiO}_{2}$ particles respectively.

\section{Preparation of optical resonators embedding Au@SiO 2 particles, references and blank samples}

Optical resonators were prepared by successive spin coating of nanocolloids of $\mathrm{SiO}_{2}, \mathrm{TiO}_{2}$ and different types of $\mathrm{Au} @ \mathrm{SiO}_{2}$ particles, following a procedure described in detail elsewhere ${ }^{20}$ and designs extracted from a computational code that simulates the optical response of a metallodielectric multilayer. Such a code is based on the method of transfer matrix and allows calculating the reflectance, transmittance and absorptance of a multilayer, as well as the spatial distribution of the electromagnetic field for a vector wave propagating through the structure. ${ }^{21}$ These sols were deposited over zero fluorescence glass (Proscitech) using a spin coater (Laurell WS-400E-6NPP) in which both the acceleration ramp and the final rotation speed could be precisely determined. The first layer was deposited using $250 \mu \mathrm{L}$ of $\mathrm{TiO}_{2}$ sol and the substrate was tilted and rotated to let the suspension cover the total glass surface. Then, the sample was accelerated up to different final speeds, to control the thickness of each layer. The final speed was chosen between nominal values of 3500 and 4500 , while the value of the acceleration was of $11340 \mathrm{rpm} \mathrm{s}^{-1}$. The spin-coating process was completed in $60 \mathrm{~s}$. Subsequently, another layer of a different type of nanoparticle was deposited, using $180 \mu \mathrm{L}$, following the same procedure. The process 
was repeated until a final number of layers forming each sample has been deposited. The thicker middle layer containing Au@SiO2 particles was built by the sequential deposition of three layers: one made of silica nanoparticles, another one of silica coated gold spheres, and a third one of silica particles. By doing so, the metal particles were eventually located in the center of the optical cavity. The layers of $\mathrm{Au} @ \mathrm{SiO}_{2}$ nanoparticles were deposited by spin-coating $100 \mu \mathrm{L}$ drops of a 1.1 wt.\% (rods) or 0.5 wt $\%$ (spheres) suspension in ethanol at a final speed of $3500 \mathrm{rpm}$, with an acceleration ramp of $8100 \mathrm{rpm} \mathrm{s}^{-1}$. The samples used as reference were deposited under identical conditions, but without the surrounding photonic crystals present in the resonator. In addition, blank samples, i.e., resonators with the same photonic properties but containing no metal particles, were built to verify the correctness of the optical characterization.

\section{Structural characterization}

Transmission electron microscopy (TEM) images of the particles were recorded with a JEOL JEM 1010 microscope operating at an acceleration voltage of $100 \mathrm{kV}$. Field emission scanning electronic microscopy (FESEM) images of cross sections of the films were taken with a Hitachi S-4800 microscope operating at $2 \mathrm{kV}$.

\section{Results and discussion}

Shown in Figure 1 are TEM images of the different types of $\mathrm{Au} @ \mathrm{SiO}_{2}$ particles used in this study, i.e., large spheres (Figure 1a) and nanorods (Figure 1b). FESEM images of the cross sections of the multilayers containing these particles are shown in Figures 1c and 1d, respectively. Backscattered electrons used to image the samples allow showing well-defined darker and lighter stripes corresponding to the (less dense) $\mathrm{SiO}_{2}$ and the (denser) $\mathrm{TiO}_{2}$ layers in the stack, as well as the exact location of the $\mathrm{Au} @ \mathrm{SiO}_{2}$ particles, whose metal cores are seen as bright spherically or rod-like shaped spots. Please note that the spin coating technique herein employed permits to place a monolayer of $\mathrm{Au} @ \mathrm{SiO}_{2}$ spheres in the middle of the defect with precision, thus a highly symmetric structure being 
attained. In the case of the rods, the deposited layer is thicker and the particles are randomly oriented. In addition, the thickness of the middle layer that behaves as an optical defect can be precisely determined, of which we take advantage to control the number and spectral position of cavity resonances. Please notice that, in a previous paper, we had demonstrated that relatively small gold particles (core diameter of $13 \mathrm{~nm}$ and shell thickness of $16 \mathrm{~nm}$ ) could be integrated in an optical resonator by the method herein employed. In that case, different absorptance spectra could be attained from the same particles by changing the refractive index of the porous layers through liquid infiltration. It remained to be proved that this method was versatile enough as to be used with particles of non-spherical shape, like the rods herein presented, or spheres of much larger size, like the over $180 \mathrm{~nm}$ diameter particles used herein. The images shown in Figure 1 evidence that the procedure proposed can be successfully used in these two cases with the only limitation of the minimum width of the optical cavity (the middle layer) that can be built, which is determined by the size of the particles that is going to host. In fact, in order to attain flat uniform layers we had to integrate the particles in optical cavities that were at least 1.5 times the size of the particle diameter. Otherwise the resulting multilayer presents bumps that would decrease the optical quality, with the risk of blurring the confinement effects we were looking for. A FESEM image illustrating this effect has been included in the supporting information.

Total reflectance $\left(R_{T}\right)$ and transmittance $\left(T_{T}\right)$ of resonator, reference and blank samples were obtained using an integrating sphere operating in the UV-Vis, attached to a spectrophotometer (Shimadzu UV2101PC). The different operational modes are described in Figure 2. From these measurements, absorptance $(A)$ was obtained as $A=1-R_{T}-T_{T}$. Figures 3 and 4 display $R_{T}, T_{T}$ and $A$ for optical resonators containing either spherical or rod-like $\mathrm{Au} @ \mathrm{SiO}_{2}$ particles, respectively. The absorptance spectra of the corresponding reference samples, made of layer of $\mathrm{Au} @ \mathrm{SiO}_{2}$ particles of the exact same thickness surrounded by thin layers of titania nanoparticles, are plotted as red dashed lines for the sake of comparison. Full optical characterization of blank samples can be found in the supporting information. In both cases, strong modifications of the absorptance are observed with respect to the reference slab. 
This has implications in the way the optical field is distributed in the multilayer. For relatively thick middle layers like the ones we had to employ to host the spherical particles, a couple of resonant modes show up instead of just one as it happens for thinner optical cavities, and this is the origin of the double peak structure observed in the absorptance spectrum displayed in Figure 3(d). The two resonances are shown explicitly in Figure 5. As mentioned before, in order to host the $\mathrm{Au} @ \mathrm{SiO}_{2}$ nanospheres, the size of the middle layer including them is such that gives rise to two resonant modes (indicated with thin vertical dashed lines), instead of the single resonant mode that would be attained with thinner optical defect slabs. ${ }^{15}$ The versatility of the proposed method is demonstrated by designing a couple of multilayered structures that present these resonances at different spectral ranges. This was achieved by adjusting the period of the photonic crystals and the thickness of the middle layer (full details are given in the caption of Figure 3). Comparing the optical response of the two ensembles, it can be readily seen that completely different absorptance is attained for $\mathrm{Au} @ \mathrm{SiO}_{2}$ nanosphere monolayers of the exact same characteristics, depending on the spectral position of cavity resonances and the photonic band gap in each resonator. While the reference presents a broad absorption peak at $\lambda \approx 600 \mathrm{~nm}$, characteristic of large gold spheres, the resonator whose optical response is displayed in Figure $3 b$ shows a much more intense and narrow peak, located at $\lambda \approx 576 \mathrm{~nm}$. In this case, the effect of the second resonant mode is minimized by forcing it to coincide with an absorption minimum $(\lambda \approx 450 \mathrm{~nm})$. For the second resonator (Figure 3d), a well-defined double peak structure with maxima located at $\lambda \approx 530 \mathrm{~nm}$ and $\lambda \approx 660 \mathrm{~nm}$ is obtained. It should be remarked that this sort of controlled modification that leads to absorptance spectra displaying localized surface plasmon peaks at different selected wavelengths, is not achievable by any other means. Also, the well-known limited capacity to absorb light of comparatively large metal spheres is herein largely increased by this combined photonic-plasmonic resonant effect.

Nanorods present two localized surface plasmon modes (transversal mode $\lambda_{T} \approx 520 \mathrm{~nm}$, longitudinal mode $\lambda_{L} \approx 840 \mathrm{~nm}$, see red dashed line in Figure $4 \mathrm{~b}$ or $4 \mathrm{~d}$ ) as a result of the polarization dependence of the extinction cross section for particles of asymmetric shape. ${ }^{22}$ In this case, the resonators were 
designed to display a cavity mode close to the transversal mode, which is typically identified as a weak and broad peak. In the multilayers, the spectral features of this absorption peak are, just as we found in the case of the spheres, largely modified: a spectrally thinner and more intense peak structure is observed. In one case, an absorption maximum occurs at $\lambda \approx 530 \mathrm{~nm}$, while in the other it peaks at $\lambda \approx 570$ $\mathrm{nm}$, further demonstrating its tunability through the design of photonic resonances. At the same time, the shape and intensity of the spectrum at those frequencies for which light is absorbed as a result of the interaction with the longitudinal plasmonic mode $(\lambda>650 \mathrm{~nm})$, are not affected by the presence of cavity resonances and hence do not present significant changes.

In order to correlate the observed effects with the phenomena taking place within the multilayer, enhancement factors, $\eta(\lambda)$, were extracted from the graphs plotted in Figure 3, by dividing the absorptance spectra of the resonator by that of the corresponding reference, and compared to the spectral and spatial dependence of the electric field intensity $|E(x, \lambda)|^{2}$, as calculated from the computer code used to design the structures herein reported. Results are shown in Figure 5. A figure describing a similar comparison for the case of the gold nanorods can be found in the supporting information. A color scale is used to indicate the field intensity at each wavelength ( $x$ axis) and distance (y axis) from the air-multilayer interface. From this comparison, it can be concluded that the observed absorptance peaks for the $\mathrm{Au} @ \mathrm{SiO}_{2}$ sphere-containing resonators are primarily due to the enhanced interaction between the embedded absorbing particles and light with wavelengths matching planar cavity resonant modes. Also, for frequencies close to the higher energy photonic band gap edge of the multilayer, the optical field is also reinforced in the defect layer. This latter phenomenon also increases absorption ${ }^{23}$ and contributes to the complex peak structure observed in the absorption spectrum of the gold particlecontaining resonators. It should also be noticed that the absorptance decreases at photonic band gap frequencies at which no resonance occurs, as a result of the blocking of incoming radiation. This comparison illustrates that spectral reinforcement or depletion of optical field intensity in planar cavities yield either stronger or weaker, respectively, matter-radiation interactions, which in turn leads to higher 
or lower probability of absorption, in this case by the embedded gold particles. These phenomena are at the origin of all effects herein reported.

\section{Conclusions}

In conclusion, we have demonstrated that the integration of metal particles in optical resonators provides a generic means to modify the optical absorption originated at localized surface plasmons. In order to prove so, relatively large particles of different shape (spheres and rods) were successfully sandwiched between two one-dimensional photonic crystals by spin coating and the effect of the interplay between the photonic resonance, which implies localization of the optical mode within the middle defect layer, and the localized surface plasmons of the particles, was thereby demonstrated. Results are explained on the basis of calculations of the spatial and spectral dependence of the optical field intensity within the multilayers. Apart from providing a new means to tailor the optical absorption of assemblies of metal particles, our results imply that special care must be taken when, in order to take advantage of field enhancement or scattering effects, metal particles are introduced in layered devices in which the optical field could be modulated by interference effects, as it may happen in organic LEDs or solar cells, ${ }^{24,25}$ dye sensitized photovoltaics, ${ }^{26,27}$ or photocatalytic slabs. ${ }^{28}$

\section{Acknowledgements}

HM thanks the Ministry of Science and Innovation for funding under grants MAT2011-23593 and CONSOLIDER CSD2007-00007, as well as Junta de Andalucía for grants FQM3579 and FQM5247.

\section{Supporting Information}

FESEM images illustrating the effect of embedding relatively large gold spheres in thin resonators, the optical reflectance and transmittance obtained for a blank sample, the comparison between the calculated electric field distribution across the gold nanorod containing multilayers and the experimentally determined enhancement factors, as well as more details on the theoretical model are included as supporting information. 


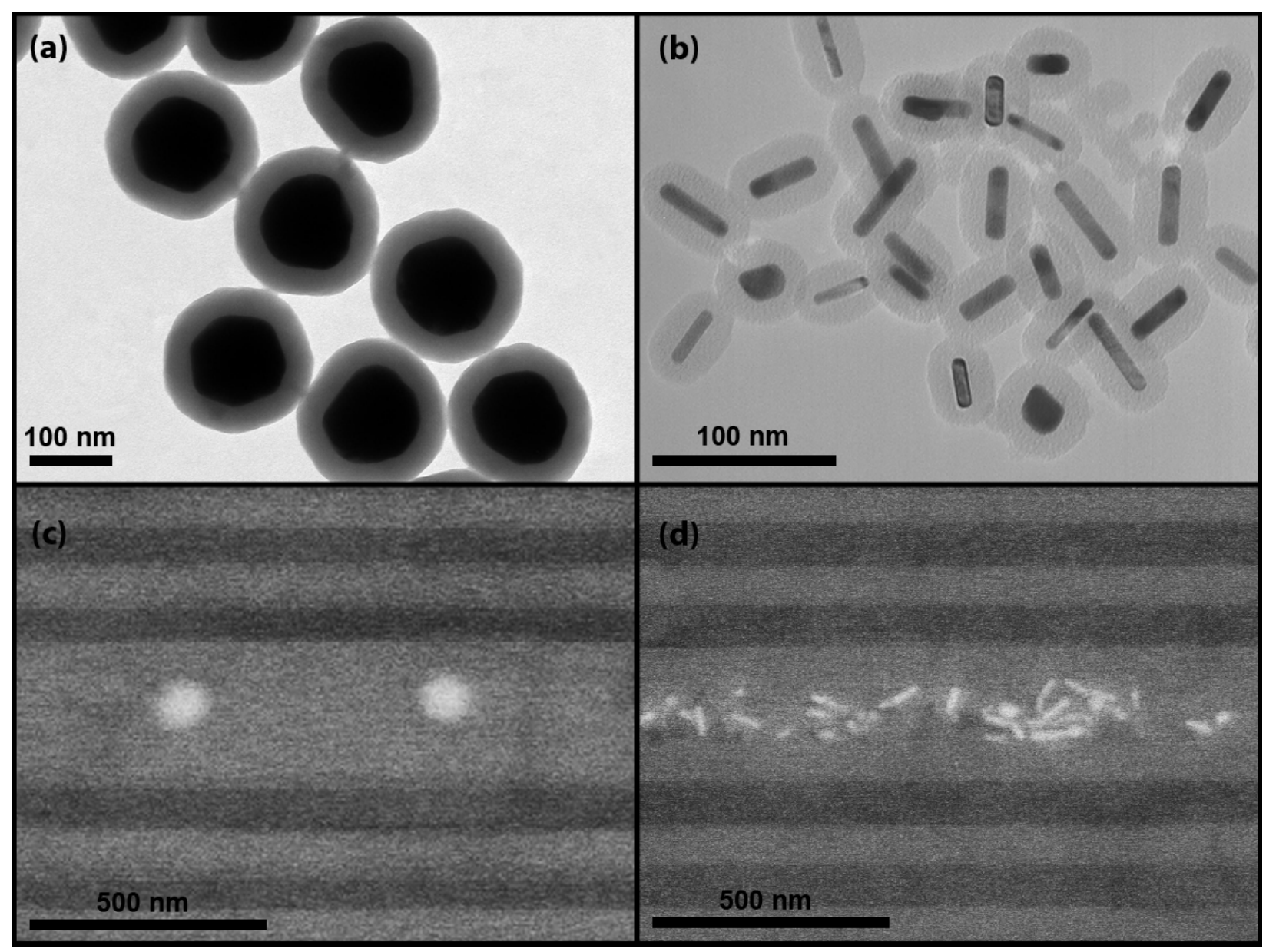

Figure 1. Top images, TEM images of $\mathrm{Au} @ \mathrm{SiO} 2$ (a) nanospheres (b) rods. Bottom, SEM backscattered electrons images of cross sections of optical resonators hosting Au@SiO2 (c) nanospheres and (d) rods. 

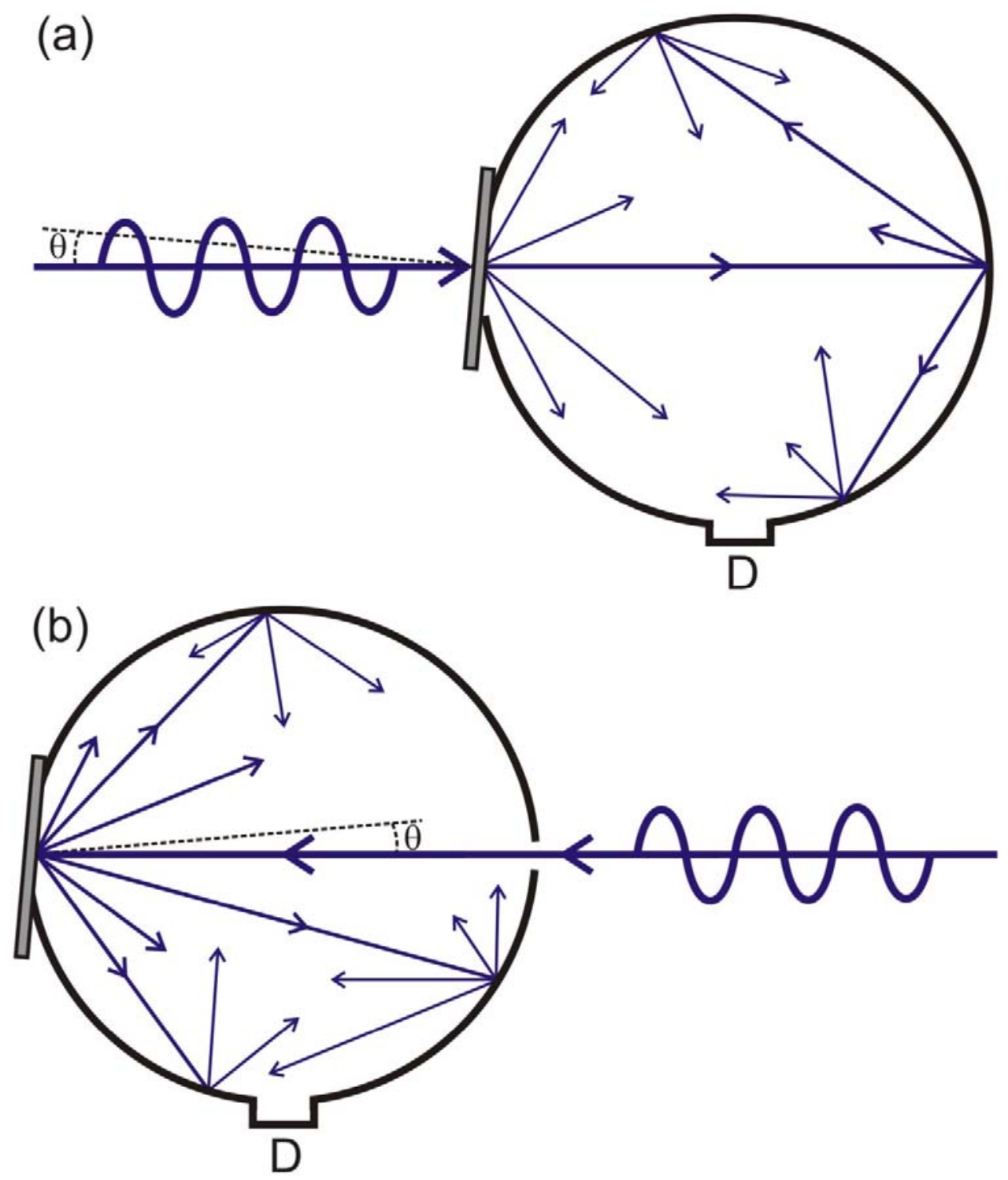

Fig 2. Experimental set-up employed to measure (a) total transmittance and (b) total reflectance. 

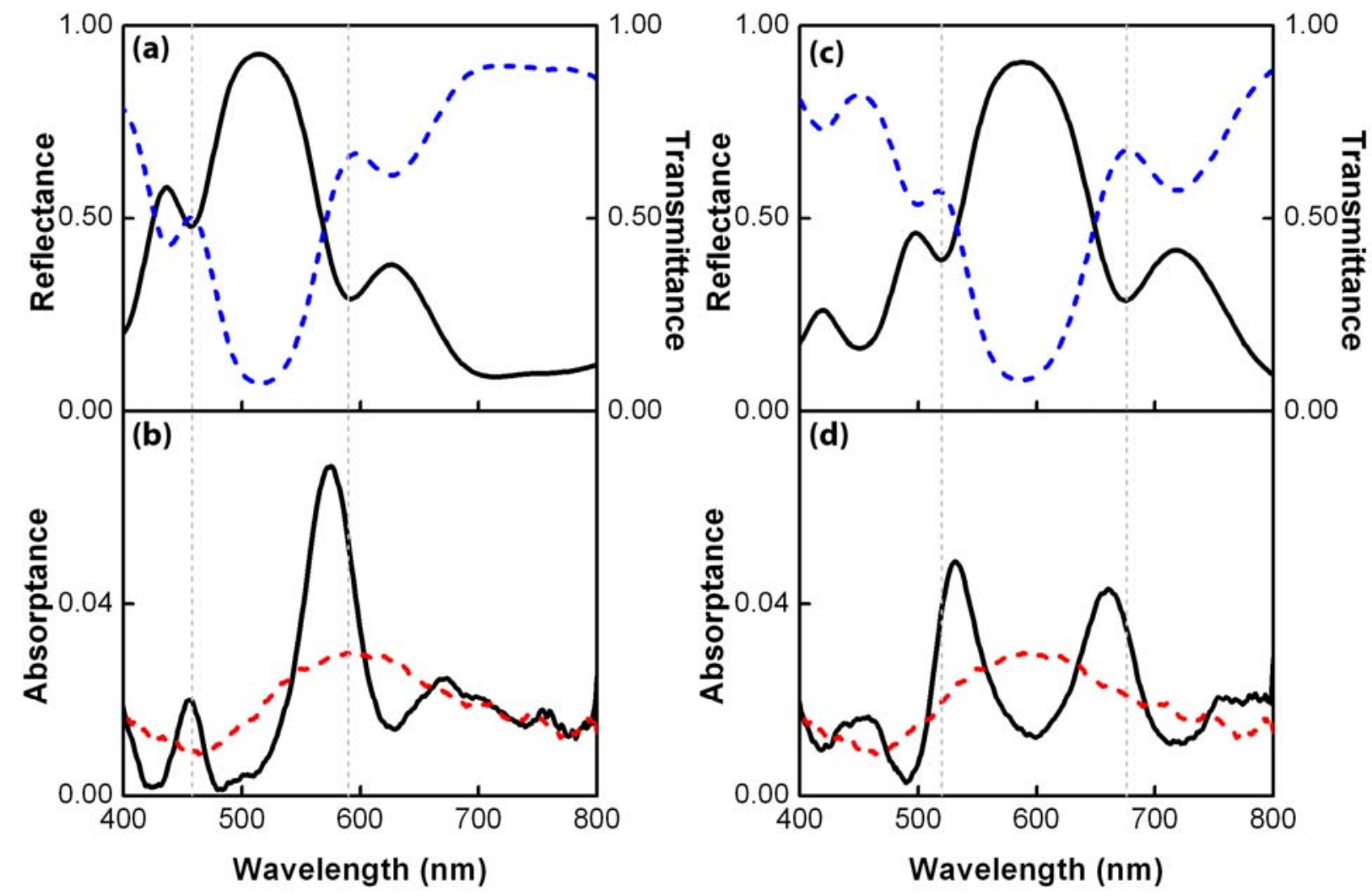

Figure 3. (a) Total reflectance (black line) and total transmittance (blue dashed line) spectra for an optical resonator made of a defect layer (total thickness $\mathrm{d}_{\text {def }}=305 \mathrm{~nm}$ ) of $\mathrm{TiO}_{2}$ nanoparticles embedding a monolayer of $\mathrm{Au} @ \mathrm{SiO}_{2}$ spheres sandwiched between two 1D photonic crystal, each one made of alternated layers of $\mathrm{SiO}_{2}$ and $\mathrm{TiO}_{2}$ of thickness $\mathrm{d}_{\mathrm{SiO} 2}=95 \mathrm{~nm}, \mathrm{~d}_{\mathrm{TiO} 2}=75 \mathrm{~nm}$. (b) Optical absorptance spectrum $\left(1-R_{T}-T_{T}\right)$ of the resonator (black line) compared to that of a monolayer of $\mathrm{Au} @ \mathrm{SiO}_{2} \mathrm{spheres}$ taken as reference (red dashed line). Same type of results are shown in (c) and (d) for another $\mathrm{Au} @ \mathrm{SiO}_{2}$ sphere containing resonator whose structural parameters are $\mathrm{d}_{\mathrm{SiO} 2}=110 \mathrm{~nm}, \mathrm{~d}_{\mathrm{TiO} 2}=85 \mathrm{~nm}$, and $\mathrm{d}_{\mathrm{def}}=$ 340. Vertical dashed lines indicate the spectral positions of the cavity resonances. 

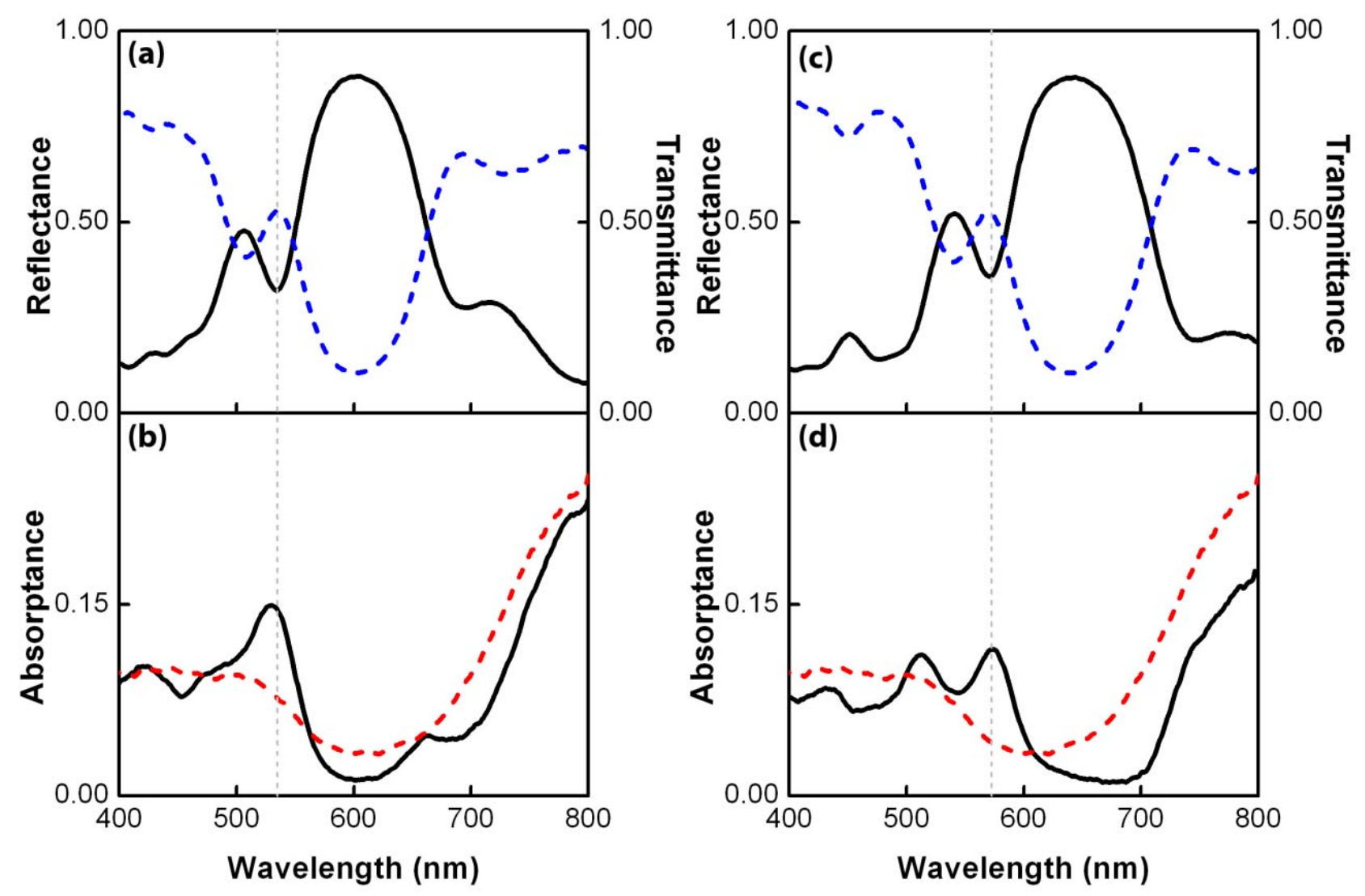

Figure 4. (a) Total reflectance (black line) and total transmittance (blue dashed line) spectra for an optical resonator made of a defect layer (total thickness $\mathrm{d}_{\mathrm{def}}=305 \mathrm{~nm}$ ) of $\mathrm{TiO}_{2}$ nanoparticles embedding a monolayer of $\mathrm{Au} @ \mathrm{SiO}_{2}$ rods sandwiched between two 1D photonic crystal, each one made of alternated layers of $\mathrm{SiO}_{2}$ and $\mathrm{TiO}_{2}$ of thickness $\mathrm{d}_{\mathrm{SiO} 2}=95 \mathrm{~nm}, \mathrm{~d}_{\mathrm{TiO} 2}=75 \mathrm{~nm}$. (b) Optical absorptance spectrum $\left(1-R_{T^{-}} T_{T}\right)$ of the resonator (black line) compared to that of a monolayer of $\mathrm{Au} @ \mathrm{SiO}_{2}$ rods taken as reference (red dashed line). Same type of results are shown in (c) and (d) for another $\mathrm{Au} @ \mathrm{SiO}_{2}$ rod containing resonator whose structural parameters are $\mathrm{d}_{\mathrm{SiO} 2}=110 \mathrm{~nm}, \mathrm{~d}_{\mathrm{TiO} 2}=85 \mathrm{~nm}$, and $\mathrm{d}_{\mathrm{def}}=340$. Vertical dashed lines indicate the spectral positions of the cavity resonances. 

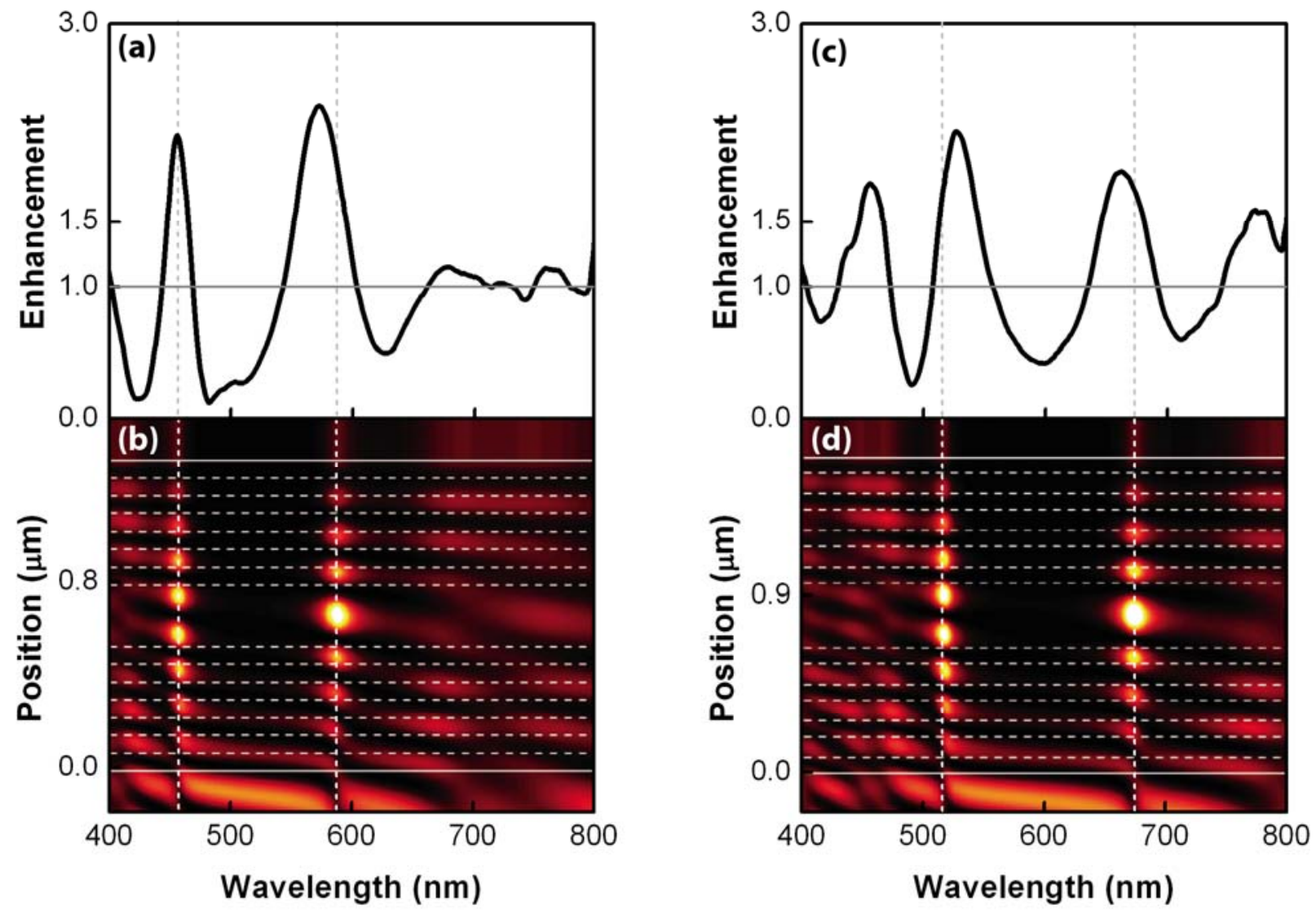

Figure 5. Absorptance enhancement factors, (a) and (c), and calculated spatial (y axis) and spectral (x axis) distribution of the square magnitude of the electric field along a cross section of the resonators, (b) and (d), for two different resonators containing a similar layer of $\mathrm{Au} @ \mathrm{SiO}_{2}$ nanospheres. Simulations were carried out considering the multilayer parameters extracted from the SEM images: (b) $d_{\mathrm{SiO} 2}=110$ $\mathrm{nm}, \mathrm{d}_{\mathrm{TiO} 2}=85 \mathrm{~nm}$ and $\mathrm{d}_{\mathrm{def}}=340 \mathrm{~nm}$; and $(\mathrm{d}) \mathrm{d}_{\mathrm{SiO} 2}=95 \mathrm{~nm}, \mathrm{~d}_{\mathrm{TiO} 2}=75 \mathrm{~nm}$ and $\mathrm{d}_{\text {deft }}=305 \mathrm{~nm}$. In both cases, refractive indexes of $\mathrm{n}_{\mathrm{SiO} 2}=1.22$ and $\mathrm{n}_{\mathrm{TiO} 2}=1.73$ were used. Horizontal dashed lines are guides to the eye to delimit the interfaces between layers. Vertical dashed lines indicate the spectral position of the cavity resonance. 
1 Nelayah, J.; Kociak, M.; Stéphan, O.; García de Abajo, F. J.; Tencé, M.; Henrard, L.; Taverna, D.; Pastoriza-Santos, I.; Liz-Marzán, L. M.; Colliex, C. Nature Phys. 2007, 3, 348.

2 Kelly, L. K.; Coronado, E.; Zhao, L. L.; Schatz, G. C.; J. Phys. Chem. B 2003, 107, 668.

3 Link, S.; El-Sayed, M. A. J. Phys. Chem. B 1999, 103, 8410.

4 Maier, S. A.; Atwater, H. A. J. Appl. Phys. 2005, 98, 011101.

5 Liz-Marzán, L. M.; Mulvaney, P. J. Phys. Chem. B 2003, 107, 7312.

6 Wang, W.; Asher, S.A. J. Am. Chem. Soc. 2001, 123, 12528.

7 García-Santamaría, F.; Salgueirino-Maceira, V.; López, C.; Liz-Marzán, L. M. Langmuir 2002, 18, 4519.

8 Wang, D. Y.; Salgueiriño-Maceira, V.; Liz-Marzán, L. M.; Caruso, F. Adv. Mater. 2002, 14, 908.

9 Inouye, H.; Kanemitsu, Y. Appl. Phys. Lett. 2003, 82, 1155.

10 Wang, D. Y.; Li, J.; Chan, C. T.; Salgueiriño-Maceira, V.; Liz-Marzán, L. M.; Romanov, S.; Caruso, F. Small 2005, 1, 122.

11 Wang, J.; Ahl, S.; Li, Q.; Kreiter, M.; Neumann, T.; Burkert, K.; Knoll, W.; Jonas, U. J. Mater. Chem. 2008, 18, 981 .

12 Yu, D.; George, M. C.; Braun, P. V. J. Amer. Chem. Soc. 2010, 132, 9958.

13 Shukla, S.; Baev, A.; Jee, H.; Hu, R.; Burzynski, R.; Yoon, Y. K.; Prasad, P. N. ACS Appl. Mater. Inter. 2010, 4, 1242.

14 Barth, M.; Schietinger, S.; Fischer, S.; Becker, J.; Nuesse, N.; Aichele, T.; Loechel, B.; Soennichsen, C.; Benson, O. Nano Lett. 2010, 10, 891.

15 Sánchez-Sobrado, O.; Lozano, G.; Calvo, M. E.; Sánchez-Iglesias, A.; Liz-Marzán, L. M.; Míguez, H. Adv. Mater. 2011, 23, 2108.

16 Rodríguez-Fernández, J.; Pérez-Juste, J.; García de Abajo, F. J.; Liz-Marzán, L. M. Langmuir 2006, 22, 7007.

17 Enüstün, B. V.; Turkevich, J. J. Am. Chem. Soc. 1963, 85, 3317.

18 Nikoobakht, B.; El-Sayed, M. A. Chem. Mater. 2003, 15, 1957.

19 Fernández-López, C.; Mateo-Mateo, C.; Alvarez-Puebla, R. A.; Pérez-Juste, J.; Pastoriza-Santos, I.; Liz-Marzán, L. M. Langmuir, 2009, 25, 13894. 
20 Colodrero, S.; Ocaña, M.; Míguez, H. Langmuir 2008, 24, 4430

21 Lozano, G.; Colodrero, S.; Caulier, O.; Calvo, M. E.; Míguez, H. J. Phys. Chem. C 2010, 114, 3681. 22 Gans, R. Ann. Phys. 1915, 47, 270.

23 Bhat, N. A. R.; Sipe, J. E. Phys. Rev. E 2001, 64, 056604.

24 Fujiki, A.; Uemura, T.; Zettsu, N.; Akai-Kasaya, M.; Saito, A.; Kuwahara, Y. Appl. Phys. Lett. 2010, 96, 043307.

25 Morfa, A. J.; Rowlen, K. L.; Reilly, T. H.; Romero, M. J.; van de Lagemaat, J. Appl. Phys. Lett. 2008, 92, 013504.

26 Ding, I. K.; Zhu, J.; Cai, W.; Moon, S. J.; Cai, N.; Wang, P.; Zakeeruddin, S. M.; Grätzel, M.; Brongersma, M. L.; Cui, Y.; McGehee, M. D. Adv. Ener. Mater. 2011, 1, 52.

27 Jeong, N.C.; Prasittichai, C.; Hupp, J.T., Langmuir 2011, 27, 14609.

28 Linic, S.; Christopher, P.; Ingram, D. B. Nature Mater. 2011, 10, 911. 